\title{
Ubiquité et discrimination des voix en centres d'appel : la quête illusoire et paradoxale d'un assureur en France et au Maghreb
}

\author{
Marie-Laure Cuisance \\ Université Paris Ouest La Défense Nanterre \\ France
}

Depuis la fin des années 1990, la filiale française d'un des leaders mondiaux du secteur de l'assurance est engagée dans un long processus de "rationalisation » de son activité. Parmi les diverses actions mises en œuvre par le management, la création de centres d'appel - d'abord en France, puis dans un pays du Maghreb - a provoqué une profonde mutation de la relation entre l'assureur et ses clients. L'objectif poursuivi par les dirigeants était le suivant: pour être plus équitables (entre les clients), plus rapides et moins coûteuses, les interactions entre l'entreprise et les clients doivent être centralisées, standardisées et contrôlées. Grâce à la combinaison des technologies du téléphone et de l'informatique, elles peuvent être déterritorialisées et réduites à un court échange entre la voix du client et celle du téléopérateur. L'interaction entre l'entreprise et le client ainsi réduite, la voix du salarié peut donc émaner de manière indifférenciée de chacun des coins de l'Hexagone, et même d'un lieu situé au-delà des frontières nationales - un centre d'appel installé en Afrique du Nord par exemple - pourvu qu'elle se fasse entendre en langue française. En installant des centres d'appel partout en France puis sur l'autre rive de la Méditerranée, les directeurs de l'entreprise font le pari de la neutralité et de l'ubiquité de la voix, celle-ci pouvant ainsi être utilisée à des fins d'efficacité économique.

Le recours aux centres d'appel par les entreprises pour traiter tout ou partie des interactions avec leurs clients est un phénomène déjà ancien qui a fait l'objet, depuis plusieurs décennies, d'une pléthore de publications montrant leur étendue géographique (Amérique du Nord, Europe, Inde, Afrique du Nord, Afrique subsaharienne, etc.), l'immense diversité des secteurs concernés (services financiers, service public, télécommunications, tourisme, milieux associatifs, etc.) et l'hétérogénéité des situations et conditions de travail. Dans une perspective plus large, le « rapport au public » a été décrit par la sociologie française comme une des scènes majeures de transformation du travail et fait l'objet d'une abondante littérature sur le quotidien des agents en contact avec la clientèle. Dans les années 1990, les « relations de services » ont ainsi placé les activités de communication entre agents et public au cœur de l'analyse, mettant en avant leur dimension coproductive (Gadrey, 2003). Par ailleurs, des questionnements sur la relation entre le langage et le travail ont progressivement émergé de travaux interdisciplinaires et ont notamment abouti, en France, à la création du Réseau Langage et Travail en 1986, réunissant initialement les sciences du travail et les sciences du langage autour d'un objet commun, les activités de langage au travail ou la "part langagière du travail » (Boutet, 2001). De nombreux travaux américains, dont ceux rattachés au courant des Workplace Studies, ont également contribué à décrire et analyser le langage et les composants communicationnels dans les situations de travail pour montrer en quoi ils contribuent à l'effectuation de l'activité de travail. L'ensemble de ces travaux a permis de montrer la pertinence d'une analyse des données langagières qui prend en considération l'organisation du travail, des relations sociales et des outils techniques pour mettre en lumière le rattachement du langage à son contexte d'action, le travail en train de se faire.

La longue histoire sociale de l'expression verbale des salariés retracée par Boutet dans La vie verbale au travail (2008) fait apparaître un tournant dans la gestion par les entreprises de la parole au travail dans les années 1970. Le langage, jusqu'alors à peine toléré et considéré comme inutile, contre-productif et illégitime - dans les usines notamment, est dès lors considéré comme une ressource naturelle qui peut être exploitée économiquement. Le modèle organisationnel des centres d'appel constitue un exemple typique de ce basculement vers un paradigme communicationnel, au centre duquel le langage devient une ressource (Habermas, 1992) ou un capital (Gumperz, 1989). Enfin, les travaux publiés sur la marchandisation du langage ont montré qu'en contexte bilingue ou plurilingue, l'instrumentalisation de la langue - dans les entreprises mais aussi le secteur touristique, par des municipalités, etc. - est indissociable des enjeux de classes et de stratification sociale dans le sens où elle participe de la reproduction des rapports de pouvoir entre les locuteurs (Duchêne, 2011; Heller, 2003; Duchêne et Flubacher, 2015).

À la suite de ces travaux et à partir de données ethnographiques produites lors d'une immersion longue dans l'entreprise ${ }^{1}$, nous poserons la question de la légitimation des voix des centres d'appel, déterritorialisées et invisibilisées, supposées être neutres et économiquement efficaces. Dirigeants, téléopérateurs et clients partagent-ils les mêmes conceptions de neutralité et d'efficacité de cet échange marchand, étiré sur les deux rives de la Méditerranée? Existe-t-il un modèle de référence partagé par l'ensemble des interactants, et si oui, comment et par qui s'impose-t-il? La description des procédés et des outils mis en œuvre par différents acteurs de l'entreprise permettra de montrer la manière dont la voix des centres d'appel est coproduite sans coprésence. L'analyse des discours de clients et de salariés sur ces 
Cuisance, Marie Laure. « Ubiquité et discrimination des voix en centres d'appel : la quête illusoire et paradoxale d'un assureur en France et au Maghreb ». Nouvelle Revue Synergies Canada, № 15 (2022)

voix et de leurs représentations mettra ensuite en évidence les nombreux indices vocaux ayant échappé à la politique de rationalisation mise en œuvre par le management. Enfin, l'analyse du traitement de ces indices, ramenés à l'origine territoriale, sociale et ethnique des téléopérateurs, à la fois par le management et les clients, dévoilera les mécanismes de discrimination et de domination qui agissent dans le cadre de l'élaboration du travail de la voix. II s'agira en somme de tenter de mettre à jour l'articulation entre normes, différences vocales et processus de légitimation et délégitimation de la voix selon des critères géographiques, sociaux et ethniques dans le contexte des centres d'appel délocalisés.

\section{La voix au cœur de l'ethnographie}

Les données mobilisées sont issues d'une ethnographie produite au début des années 2010 dans le cadre de mon doctorat en anthropologie sur les mutations de la relation assureur-assurés (Cuisance, 2016). Ce travail s'est appuyé sur une " observation participante » de près de trois années au sein d'un groupe d'assurance français avec lequel j'étais liée par un contrat CIFRE (Convention Industrielle de Formation par la Recherche), un dispositif français financé par le ministère de l'Enseignement supérieur et de la Recherche qui permet la collaboration entre un doctorant, une entreprise et un laboratoire de recherche public pour la réalisation d'une thèse. Ma position d' " anthropologue » salariée de la " direction du service client» a ainsi permis l'observation, "de l'intérieur », du travail quotidien des acteurs de l'entreprise (directeurs, managers, téléopérateurs, agents d'assurance, etc.) dans les bureaux et centres d'appel en France et au Maghreb, la participation aux différents projets de rationalisation des interactions avec la clientèle et l'accès à plusieurs centaines d'enregistrements, effectués par l'entreprise, des conversations entre les téléopérateurs et les clients. Une campagne d'entretiens semi-directifs menés hors des murs de l'entreprise avec vingt-sept clients de la compagnie d'assurance complète ce travail ethnographique. L'ensemble de ces données a permis de saisir la manière dont la création des centres d'appel a produit un bouleversement du rapport des acteurs (assureur et assurés) au temps, à l'espace, à l'Autre. Leur relecture permet d'explorer la notion de voix en centre d'appels à travers une analyse de son langage, de sa musicalité et de ses représentations mais aussi des actes et des discours de ceux qui la produisent, la contraignent, la contrôlent ou l'écoutent et au prisme des différents lieux d'où elle émane et où elle est entendue et interprétée.

\section{L'élaboration de la voix : une coproduction sans coprésence}

Jusqu'alors, lorsqu'ils étaient victimes d'un incident (fuite d'eau, incendie, cambriolage, etc.) ayant provoqué des dégâts sur leur lieu d'habitation ${ }^{2}$, les clients de l'entreprise étaient invités à se rendre physiquement à l'agence dans laquelle ils avaient souscrit leur contrat - proche de leur domicile le plus souvent - ou encore à adresser un courrier à leur agent d'assurance afin d'obtenir éventuellement l'indemnisation de leur préjudice. Quel que soit leur lieu d'habitation, les assurés sinistrés sont désormais invités à ne plus se déplacer mais à appeler un numéro unique pour « déclarer leur sinistre ». Bien que le centre d'appels soit une forme d'organisation largement banalisée - les clients de l'entreprise étant régulièrement en contact avec d'autres téléopérateurs employés dans de nombreux autres secteurs d'activités - sa mise en place a bouleversé la relation entre l'entreprise et ses clients. Le règlement des "sinistres ", qui faisait autrefois partie intégrante du "métier » d'assureur, incarné par la présence physique des nombreux agents d'assurance répartis sur l'ensemble du territoire français, fait désormais l'objet d'interactions sans face-à-face, réduites à un échange téléphonique.

Les centaines d'appels quotidiens reçus par l'entreprise font l'objet d'un premier tri selon la nature de la demande (ouverture ou suivi d'un dossier par exemple) puis sont répartis de manière aléatoire entre les salariés disponibles des différents centres d'appel de l'entreprise. Lors de la création, dans les années 1990, de ses centres d'appel situés dans la périphérie de cinq villes françaises, l'entreprise a essentiellement fait appel à des salariés déjà présents dans l'entreprise auxquels elle a proposé une reconversion professionnelle, de manière à éviter des licenciements consécutifs à la disparition de certains emplois - dus à l'informatisation croissante des activités notamment. Si le pourcentage de femmes est légèrement supérieur à celui des hommes, leurs profils sociologiques (âge, origine sociale, niveau d'étude, etc.) sont extrêmement variés. Parmi ceux que j'ai rencontrés, un ancien salarié du « service courrier », âgé de quarante-huit ans, n'ayant « pas fait d'études [supérieures] ", m'expliquait que de la « distribution du courrier dans les services " à la " gestion des clients », " le chemin avait été long », qu'il avait dû "travailler dur », mais que ce nouvel emploi de téléopérateur constituait une véritable « promotion » dont «sa femme [était] fière ». À l'inverse, pour une ancienne collaboratrice âgée de trentecinq ans ayant travaillé plus de quinze années dans une agence d'assurance, titulaire d'un « BTS » ${ }^{3}$ en assurances, le travail en centre d'appels était plutôt perçu comme dévalorisant (Cuisance, 2016). Ces hommes et ces femmes composent des équipes d'une dizaine de salariés, placés sous l'autorité directe d'un manager et installés, à proximité les uns des autres, à des postes de travail situés dans des espaces 
Cuisance, Marie Laure. « Ubiquité et discrimination des voix en centres d'appel : la quête illusoire et paradoxale d'un assureur en France et au Maghreb ». Nouvelle Revue Synergies Canada, № 15 (2022)

ouverts. Pour effectuer leurs tâches quotidiennes, ils disposent chacun d'un ordinateur relié à un téléphone équipé d'un casque, ainsi que de nombreux documents.

Au début des années 2000 , soit une dizaine d'années après la création des premiers centres d'appel et face à l'augmentation du nombre d'appels et de dossiers à traiter, l'entreprise franchit un nouveau cap dans la déterritorialisation des interactions avec sa clientèle. Comme bon nombre d'entreprises françaises (Kettani et Peraldi, 2011), elle installe un de ses centres d'appel dans un pays d'Afrique du Nord, profitant des nombreux avantages « concurrentiels » qu'offre la présence abondante sur le marché du travail d'une population jeune, nombreuse, au niveau d'éducation élevé, maîtrisant la langue française (ayant suivi des études dans un système éducatif largement francophone et inspiré du système français) et en échange de salaires nettement inférieurs à ceux pratiqués en France. À cela s'ajoutent les progrès informatiques qui permettent des communications accélérées sur de longues distances et le développement massif, dans les pays du Sud, d'infrastructures gigantesques et modernes pouvant accueillir les centres d'appel étrangers, encouragé par les États en quête de développement économique. Plus d'un millier de voix nord-africaines se joignent aux voix françaises pour répondre aux clients de l'entreprise. Jeunes hommes et femmes, récemment diplômés et occupant, pour une grande partie d'entre eux, leur premier emploi, les téléopérateurs nord-africains forment une population plus homogène que leurs collègues français. Ils sont salariés de l'entreprise et les tâches qui leur sont confiées sont similaires à celles des salariés français. Néanmoins, leurs conditions de travail diffèrent sensiblement. Leur espace de travail est plus restreint, leurs salaires sont nettement inférieurs et, contrairement à ceux des salariés français qui sont fixes, ils varient selon la "qualité » de leur travail qui est évaluée à partir de l'enregistrement de leurs conversations avec les clients. Ils disposent de moins de jours de congés payés, leurs journées de travail sont plus longues et la surveillance à laquelle ils sont soumis (calcul du nombre de tâches effectuées, de la durée des appels, etc.) est plus intense.

Le travail des téléopérateurs de l'entreprise consiste essentiellement au traitement d'appels « entrants », émanant des clients de l'entreprise ayant subi un dommage ${ }^{4}$. Cette activité est multimodale, dans le sens où elle s'effectue par téléphone et est organisée, assistée et contrainte par différents dispositifs techniques et informatiques. Le cadre organisationnel et technique a donc été mis en place en amont des interactions entre le client et l'entreprise, par des salariés du siège du groupe (cadres, chefs de projets, informaticiens, etc.) - ou encore des consultants externes - dont le travail consiste à organiser et superviser l'activité des centres d'appel. La voix des téléopérateurs est aussi soumise à des injonctions permanentes qui prennent la forme de formations, de campagnes de sensibilisation ou encore de programmes d'amélioration, élaborés également par les directions centrales - souvent la direction des ressources humaines - dont le but affiché est l'amélioration du service rendu au client. En plus d'être régulièrement « notées » par les managers, les conversations entre téléopérateurs et clients sont également évaluées par d'autres catégories de salariés, dont le travail consiste pour certains à «contrôler » leur qualité, d'autres à « piloter » l'activité, ou pour d'autres encore à « mesurer » la satisfaction du client. Pour espérer obtenir de « bons » résultats - individuellement ou collectivement - les téléopérateurs tentent donc de se conformer au mieux à la multitude et l'hétérogénéité des critères de surveillance de leur voix établis par ces nombreux contrôleurs (tels que l'obligation de se présenter nominativement, de montrer de l'empathie, de reformuler la demande du client, d'éviter de le faire patienter, de proposer des services adaptés, etc.).

À l'intérieur de ce cadre normatif contraignant, les téléopérateurs doivent en outre s'adapter constamment aux demandes, incompréhensions ou inquiétudes exprimées par le client et aux imprévus ou malentendus qui surviennent durant la conversation. II n'est pas rare, par exemple, que suite à l'énoncé par le téléopérateur du "type de sinistre », c'est-à-dire de son diagnostic - une "infiltration par façade » ou encore une "débordement d'appareil à effet d'eau " par exemple, le client demande à être éclairé sur la signification des termes employés et sur les conséquences attendues. De même, le salarié peut être amené, en plus de lui expliquer les mesures prises, à tenter de rassurer tel assuré victime d'un cambriolage par exemple, ou à essayer d'apaiser le mécontentement de tel autre dont le paiement d'une indemnisation tarderait à être effectué. La communication entre les deux acteurs audibles nécessite donc des ajustements permanents de la parole du téléopérateur, qui met en œuvre ses compétences techniques, sociales et rhétoriques pour s'adapter au contexte et aux compétences propres du client, tout en veillant à ne pas déborder du cadre imposé par l'entreprise. La conversation en centre d'appel n'est donc pas une interaction entre deux participants mais, pour reprendre les termes de Goffman (1974), entre un « praticien-réparateur » $A$ (le salarié), un « propriétaire » B (le client) et un " objet » C (l'incident ayant provoqué des dommages éventuellement couverts par le contrat d'assurance). La voix audible du « praticien-réparateur » apparaît en outre comme l'agencement de plusieurs voix qui parlent à travers lui, souvent contradictoires puisque poursuivant des buts différents, qui l'enjoignent à la neutralité, l'efficacité, la productivité, et l'empathie, la satisfaction des demandes du client tout à la fois. Puisqu'il s'appuie pour une large part sur les technologies du téléphone et de l'informatique, ce travail complexe de coproduction s'effectue sans face-à-face, les différents acteurs de la relation se trouvant à distance les uns des autres. En effet, si les clients ne rencontrent jamais les téléopérateurs, il en est de même pour les salariés 
Cuisance, Marie Laure. « Ubiquité et discrimination des voix en centres d'appel : la quête illusoire et paradoxale d'un assureur en France et au Maghreb ». Nouvelle Revue Synergies Canada, No 15 (2022)

responsables de l'organisation et du contrôle de leurs activités. Situés au siège de l'entreprise, ils sont très peu nombreux à s'être déjà rendus sur l'une ou l'autre des plateformes téléphoniques françaises ou nordafricaine, dont l'accès est très restreint. II en résulte une situation dans laquelle de nombreux acteurs, impliqués dans un contexte de communication complexe, ne partagent à aucun moment le même environnement et se trouvent dans l'impossibilité de saisir les indices non-verbaux de l'interaction, à travers les objets, les regards, ou encore l'hexis corporelle. La voix des centres d'appel se caractérise ainsi à la fois par l'agencement médiatisé de différents langages et par une disjonction de sa dimension verbale et non verbale, phénomène qu'une cliente française que j'ai interrogée lors d'un entretien ethnographique exprimait ainsi : "On a toujours une voix plus ou moins lointaine, absente, comme quelqu'un qui n'est pas vraiment là ».

\section{Interpréter des signes de la voix : les impensés de la standardisation}

Lorsque mon enquête ethnographique débute en 2010, une partie des appels téléphoniques est déjà reçue par les salariés nord-africains. Leur nombre a connu une augmentation rapide durant les mois précédant mon arrivée dans l'entreprise pour atteindre plusieurs dizaines de milliers par an. À ce momentlà, les directeurs français en charge de la supervision et de la répartition de l'activité entre les centres d'appel français et nord-africain considèrent que, du point de vue «technique » et financier, la délocalisation d'une partie de l'activité est un succès. Pourtant, dans le même temps, de nombreuses " réclamations " de clients, d'agents généraux affluent au siège de l'entreprise et les " enquêtes de satisfaction » menées par le service marketing auprès de clients ayant été en contact avec les centres nord-africains montrent un "mécontentement nettement significatif ", comparé à ceux ayant été en contact avec les téléopérateurs situés en France. Les dirigeants craignent de devoir affronter un nombre croissant de résiliations des contrats par leurs clients, le refus des agents généraux de coopérer, ainsi que la colère des salariés français. Malgré les efforts déployés par l'entreprise pour parler à ses clients d'une seule et même voix, outillée et standardisée, une distinction entre les téléopérateurs français et maghrébins demeure dans les discours produits par les clients ou au siège de l'entreprise, par les salariés responsables de l'organisation de l'activité des centres d'appel.

Préoccupation majeure des managers du siège de l'entreprise, cette dissonance entre les voix françaises et les voix nord-africaines occupe une large place dans les discours que j'ai pu entendre entre les murs de l'entreprise. Pour expliquer l'échec de l'entreprise à faire chorus, les particularités des salariés nordafricains sont passées au crible et incriminées, à partir des « plaintes » de certains clients :

Alors, ce que [les clients] disent, c'est [que] les collaborateurs [maghrébins] ne parlent pas bien français, qu'ils ne comprennent pas les noms (...), qu'ils sont lents. Soi-disant ils sont pas sympas, ou ils ont rien compris, ou alors ils ont un accent tellement fort qu'on comprend pas ce qu'ils disent, bref, y'a toujours un truc. La liste est longue! (Extrait d'un entretien ethnographique ${ }^{5}$ mené avec un directeur français de l'entreprise, responsable de la supervision et de la coordination de l'ensemble des centres d'appel)

Parmi les nombreux griefs, le « niveau de français » est jugé « faible » par certains. Pourtant, ce critère est déterminant lors de l'embauche des salariés maghrébins qui ont suivi des études universitaires en français - généralement cinq années d'études après le Baccalauréat. Certains salariés français du siège de l'entreprise me font néanmoins la démonstration d'un manque de maîtrise des "subtilités " de la langue française. Bien qu'ils ne soient jamais en contact avec leurs collègues maghrébins, ils puisent souvent dans une réserve restreinte d'anecdotes supposées «typiques " des erreurs commises de l'autre côté de la Méditerranée, qui circulent activement dans l'entreprise, mais dont la véracité n'a jamais pu être démontrée. En voici un exemple qui m'a été rapporté dès mon arrivée dans l'entreprise par un jeune salarié ayant participé, depuis le siège de l'entreprise, à l'élaboration d'un programme de formation destiné aux téléopérateurs. Bien que n'ayant jamais été en relation directe avec les salariés maghrébins, il souhaitait me « donner une idée » des « incompétences » en matière de « communication » :

Y'a des erreurs grossières qui font désordre quand même. Par exemple, ils ne savent pas ce qu'est un nid-de-poule. Donc, ils pensent que l'accident a été provoqué par une poule. Ou dans l'Est [de la France], les ralentisseurs, ils appellent ça des «gendarmes couchés». Ça non plus [les collaborateurs maghrébins], savent pas. Donc, ils pensent qu'il y a un blessé. Y'a plein de trucs comme ça qui passent pas. (Extrait d'un entretien ethnographique mené avec un manager de la direction du service client au siège francilien de l'entreprise)

À ces récriminations basées sur des indices de nature segmentale au sujet de l'emploi incorrect ou inexact de mots ou d'expressions s'ajoutent parfois d'autres dissonances, comme l'illustre l'extrait suivant : 
Cuisance, Marie Laure. « Ubiquité et discrimination des voix en centres d'appel : la quête illusoire et paradoxale d'un assureur en France et au Maghreb ». Nouvelle Revue Synergies Canada, № 15 (2022)

II y a quelque chose d'indéfinissable. Je ne saurais même pas dire quelle phrase ou quel mot exactement. Quelque chose qui fait penser qu'on n'est pas en France. Je ne sais pas, même dans la politesse, c'est trop ou c'est feint, je ne sais pas. Ou c'est des trucs qu'on ne dirait pas, ou... en tout cas pas comme ça en France. Mais c'est pas évident, parce que c'est pas non plus... C'est pas si flagrant, mais c'est des détails, une prononciation, un ton qui sonne pas juste, qui te met la puce à l'oreille. (Extrait d'un entretien ethnographique mené avec un client français à son domicile à Paris)

Ce discours souligne la difficulté d'objectiver ces indices supra-segmentaux, présentés comme des "petites choses », " indéfinissables », qui peuvent néanmoins "mettre la puce à l'oreille » et "tout changer ». Bien que les dirigeants français s'y emploient avec acharnement, cherchant du côté des chiffres, des enquêtes de satisfaction, du « feeling » des salariés français, ils peinent à objectiver ce relatif échec :

(...) C'est vrai qu'on a des mécontentements, je ne peux pas le nier. Mais c'est difficile de faire la part des choses entre une sorte de mauvaise foi de la part de certains clients, agents, salariés (...). C'est pour ça que j'essaie d'avoir des numéros de dossier, des exemples précis, mais on n'arrive pas forcément à avoir des exemples concrets, du factuel. (Extrait d'un entretien ethnographique mené avec un directeur français au siège de l'entreprise)

Partant du postulat selon lequel la maîtrise de la langue française et l'acquisition, par une formation technique, d'un langage propre au domaine de l'assurance suffiraient à garantir l'efficience des voix nordafricaines, aucun programme destiné à neutraliser l'accent des salariés, ou encore à l'apprentissage d'un " savoir-être » ou de la « culture » (française) n'a été mis en place, contrairement à d'autres entreprises utilisant des centres d'appels, en Inde notamment (Cowie, 2007). De même, aucun dispositif d'observation, de contrôle ou de mesure de ces indices verbaux n'a été mis en place. Ne pouvant être mesurés, les indices que constituent le ton de la voix, son accent, ses spécificités langagières, ne figurent jamais dans les discours et documents officiels de l'entreprise, tels que les rapports d'activité. Aux prises avec les paradoxes de leur propre idéologie de neutralité, les dirigeants de l'entreprise se trouvent dans l'incapacité d'écouter l'ensemble des indices d'une voix qu'elle a ardemment souhaité réduire à sa seule efficacité économique.

Difficiles à objectiver - et donc à prendre au sérieux - par le management, les griefs concernant les caractéristiques vocales des salariés nord-africains leur sont rarement adressés directement. Néanmoins, en plus des normes imposées explicitement par le management, de nombreux salariés régulent euxmêmes leurs pratiques vocales, mettant en place des stratégies d'évitement ou d'adaptation lorsqu'ils se sentent en difficulté ou pour mieux se soumettre aux exigences supposées de l'entreprise et de la clientèle. Ils peuvent par exemple demander aux clients le code postal de leur lieu d'habitation qu'ils retrouvent aisément sur internet, de façon à ne pas risquer de lui faire épeler ou répéter un nom propre qui serait connu de tous les Français. Ils apportent également des changements dans l'emploi du vocabulaire. Par exemple, certains d'entre eux m'ont précisé qu'ils réservent désormais l'usage du mot « lavabo » aux discussions concernant la salle de bain, alors qu'auparavant, ils l'employaient également pour désigner l' " évier » de la cuisine, ce qui suscitait parfois des malentendus avec les clients. À force d'écouter les clients, ils disent aussi modifier la manière de prononcer certains mots, le ton des formules de politesse qui leur sont imposées par l'entreprise. D'autres salariés m'ont encore expliqué qu'ils prenaient soin de consulter chaque jour les données météorologiques de la France de manière à pouvoir évoquer « le temps qu'il fait ", ayant " remarqué que les Français aimaient bien parler de la pluie et du beau temps », ou encore qu'ils évoquaient fréquemment les fêtes "françaises" (Noël par exemple), pour faciliter l'interaction. C'est donc pour réduire l'inconfort lié à la tension qui s'exerce sur eux que les téléopérateurs inventent, en situation, des pratiques concrètes et quotidiennes destinées à compenser leur précarité langagière.

En tentant de réduire la voix de ses téléopérateurs, non seulement à ses caractéristiques verbales mais, plus encore à son efficience technique, l'entreprise a largement négligé ses nombreux autres indices, de l'emploi de certains mots à la musicalité de la voix. Or, en l'absence de contact visuel entre les interactants, ces indices revêtent d'autant plus d'importance qu'ils ne peuvent être corrigés par l'observation des gestes ou des regards. À ce stade de la réflexion, l'idéologie de neutralité, d'ubiquité et d'efficacité de l'entreprise, selon laquelle « le client, d'où qu'il appelle et où qu'il soit reçu [dans un centre d'appel de l'entreprise en France ou au Maghreb] doit être traité de la même manière » - pour reprendre les mots de ses dirigeants - est mise à mal. La marchandisation et la déterritorialisation de ses voix, si elle permet de donner en partie l'illusion d'une homogénéité en rendant invisibles ses salariés et leur lieu de travail, ne permettent aucunement de réduire leur voix à la seule efficience économique par des procédés discursifs contraints. Tout au plus, elle permet aux décideurs d'en ignorer temporairement la dimension métalinguistique. 
Cuisance, Marie Laure. « Ubiquité et discrimination des voix en centres d'appel : la quête illusoire et paradoxale d'un assureur en France et au Maghreb ». Nouvelle Revue Synergies Canada, № 15 (2022)

\section{Discriminer : les espaces géographiques, sociaux et politiques de la voix}

Alors que quelque chose semble résister autant à l'organisation, au contrôle, qu'à l'énonciation, un des managers français avance une raison censée expliquer la concentration des critiques à l'endroit des salariés maghrébins :

Nous avons des centres d'appel dans toutes les régions de France et [au Maghreb]. On entend des plaintes sur l'accent des collègues [nord-africains] mais jamais sur l'accent du nord, celui du sudouest, ou celui de la banlieue parisienne ! C'est quand même bien que c'est de la mauvaise foi !. (Un manager français)

Ce qu'il désigne par le terme euphémisé de « mauvaise foi » apparaît comme une discrimination faite par certains salariés, cadres, dirigeants ou clients français, qui s'apparente à un phénomène de glottophobie, qu'Arditty et Blanchet (2008) définissent comme « une forme particulière de l'altérophobie appliquée à des pratiques linguistiques autres, stigmatisées en elles-mêmes, pour elles-mêmes, et avec elles les personnes et les groupes qui les parlent et qui s'y identifient, personnes et groupes dont les capacités humaines au langage sont dès lors réputées sous développées ". Au sein de la diversité des voix émanant des différents centres d'appel, les différents parlers ne font pas tous l'objet d'une stigmatisation ${ }^{6}$ mais la voix nord-africaine, réifiée, est décrite dans les discours glottophobes recueillis comme présentant un écart à la norme qui nuirait à la qualité de l'interaction entre l'entreprise et ses clients. Ces discours opèrent une distinction entre deux voix s'exprimant en langue française. L'une est jugée légitime, regroupant la diversité des parlers pouvant être identifiés comme hexagonaux. L'autre, unifiée - la façon de parler dans ce pays du Maghreb - est présentée comme étant illégitime. Une normalisation linguistique s'impose, régissant les conversations avec les clients français et dont les normes sont implicites puisque dans la sélection des salariés, leur formation et leur évaluation, il n'est jamais fait mention de critères ouvertement discriminants tels que l'accent.

Malgré la volonté des dirigeants de l'entreprise de passer outre les frontières nationales pour maximiser ses profits, les données ethnographiques recueillies dans ces centres d'appel tendent à montrer que l'émission et la réception des voix restent tributaires des espaces sociaux, même invisibilisés, desquels elles émanent. La discrimination linguistique qui s'opère dans les discours analysés semble largement conditionnée par les représentations des lieux où sont produites les voix des téléopérateurs. L'extrait suivant donne une illustration de l'imaginaire des centres d'appel nord-africains véhiculé par la voix :

Tu te dis, on parle de ma salle de bain, je suis dans mon salon en train de parler [au téléopérateur] et lui, il est je sais pas où en Afrique du Nord. Et là, tu as les images complètement stéréotypées qui te viennent à l'esprit, sur l'Afrique et autres. Alors que bon, ces gens-là, ils sont pas non plus en train de manger du couscous dans leur tente dans le désert, pas du tout! D'ailleurs, ils ont dû faire des études, à l'étranger peut-être, ils doivent être dans des capitales super modernes. Mais qu'on le veuille ou non c'est assez troublant et du coup tu doutes, voire même t'as pas confiance. (Extrait d'un entretien ethnographique mené avec un client français à son domicile en banlieue bordelaise)

Cette voix, qui serait "reconnaissable », apparaît comme un puissant vecteur, voire une caisse de résonance des représentations d'un ailleurs fantasmé, exotisé et souvent déprécié - du Sud, de l'Orient, de l'Afrique. À travers la voix, le client - tout comme l'ensemble de ceux qui sont en interaction avec les téléconseillers nord-africains - fait l'expérience concrète et quotidienne de la globalisation des échanges. Le temps d'une conversation, le client français et le téléopérateur maghrébin sont réunis dans une salle de bain parisienne ou un salon alsacien, autour des dégâts provoqués par une fuite d'eau ou un incendie par exemple. Néanmoins, la mondialisation - qui concerne aussi les pays du Sud - n'abolit pas totalement les frontières, ni l'altérité. Les voix nord-africaines de l'entreprise, entendues sur le territoire français, sont stigmatisées, renvoyées au lieu d'où elles sont émises et à ses représentations stéréotypées.

La construction de ces stéréotypes peut également emprunter au contexte social et économique, comme en témoigne le discours d'une téléopératrice française de l'entreprise que j'ai pu recueillir. Employée d'un des centres d'appel de l'entreprise situé en France, elle dit ne pas « accepter » de devoir « partager » son activité professionnelle avec des salariés nord-africains, avec lesquels elle doit parfois coopérer sur certains dossiers.

Alors moi, j'ai rien contre les petits jeunes, nos collègues [maghrébins], pas personnellement, faut pas être raciste quand même. Mais moi, je suis pas d'accord. On a pas de boulot ici en France. Moi, j'ai été embauchée ici à une période où y'avait du travail. C'était une boîte qui embauchait et où t'avais la sécurité de l'emploi. Mais maintenant c'est plus ça ! Et le travail qui part [au Maghreb]. Excuse-moi, mais moi je trouve pas ça normal. Alors ces gens [les collaborateurs nord-africains], 
Cuisance, Marie Laure. « Ubiquité et discrimination des voix en centres d'appel : la quête illusoire et paradoxale d'un assureur en France et au Maghreb ». Nouvelle Revue Synergies Canada, № 15 (2022)

moi, j'ai rien contre eux, c'est pas de leur faute en plus et tant mieux pour eux s'ils ont du boulot, mais moi j'ai du mal. On a beau nous dire que c'est des collègues, qu'on est dans le même bateau et qu'on travaille main dans la main, moi j'ai du mal. (...) C'est quand même, il faut dire le mot, c'est quand même du travail qui pourrait être chez nous et qui part [au Maghreb]! Dans la période actuelle, c'est quand même... C'est compliqué. (...) Et surtout pour être moins bien fait, il n'y a qu'à les écouter pour s'en rendre compte. (Extrait d'un entretien ethnographique mené avec une téléopératrice française en région parisienne)

Ce type de discours n'est pas majoritaire dans l'entreprise. II mobilise des éléments tronqués du contexte économique français (un chômage persistant) et politique (des dispositions facilitant la délocalisation à l'étranger) pour reconstruire une frontière entre " eux » et " nous », soit instaurer une polarisation sociale négative des deux espaces, basée sur des préjugés de race. Dans d'autres discours - ceux de certains managers français notamment, les préjugés raciaux ne sont pas présentés comme négatifs mais comme des « compétences ", servant de justification à la délocalisation de l'activité, comme en témoigne l'extrait suivant :

Les téléopérateurs maghrébins ne sont pas comme les français, ils ont envie de travailler! Ils ne rechignent pas comme en France s'il faut faire des heures supplémentaires. (...) Ils sont plus disciplinés (...) Ils râlent moins, ils ne contestent pas les consignes. (...) Ils ne réclament pas une pause syndicale toutes les cinq minutes. (Extrait d'un entretien ethnographique mené avec un manager français employé au Maghreb)

Selon ce manager, les compétences langagières négatives des salariés maghrébins seraient largement compensées par des compétences « positives » (l'appétence au travail et une soumission plus grande à la hiérarchie), justifiant ainsi l'élargissement du marché de la voix au-delà des frontières nationales. Ces discriminations raciales s'ajoutent aux stéréotypes de classe qui s'appliquent aux téléopérateurs, comme en témoigne l'extrait suivant:

Tous ces petits jeunes [les salariés des centres d'appel]... Vous savez mieux que moi je pense mais, les pauvres, ils font de leur mieux je crois ! (...) Ils doivent être un peu exploités, mal payés... Pfff, c'est pas un métier, hein! (Extrait d'un entretien ethnographique mené avec un client français à son domicile à Besançon)

À l'intérieur de cette catégorie des « travailleurs du langage » (Boutet, 2008) ou encore « nouveaux OS du tertiaire » (Buscatto, 2002), souvent perçue de manière hétérogène comme peu qualifiée et " exploitée » économiquement, s'ajoute une classe des téléopérateurs nord-africains encore davantage infériorisée du fait de son appartenance ethnique et sociale. Ces discriminations langagières liées à l'appartenance sociale ou raciale, qu'elles servent de prétexte au rejet ou de justification à l'élargissement des murs de l'entreprise, sont toujours indirectes. Les propos à caractère racial, voire raciste, que j'ai pu recueillir dans le discours de certains salariés, cadres ou clients, ont été entendus dans le cadre d'entretiens ethnographiques ou de discussions informelles menés le plus souvent hors de l'entreprise. Souvent euphémisés, ils étaient toujours accompagnés de justifications se défendant d'un racisme qui serait répréhensible.

Selon la consigne du management - et sans qu'il soit permis de douter des intentions ni de la bonne foi de la majorité de ses représentants, les collaborateurs nord-africains « sont considérés comme des collègues exactement comme les autres ». Pourtant, les mécanismes de différenciation visant, dans le cadre de l'activité de l'entreprise, à la légitimation de certains parlers et à la délégitimation de certains autres, peuvent être reliés à des enjeux de domination basés sur des critères sociaux et raciaux. Sans dire leur nom, ils agissent sans être réellement combattus, puisque largement ignorés, impensés. Dans l'entreprise, la voix apparaît ainsi comme un des critères de discrimination socialement acceptés, à propos duquel Woolard (1998, p. 19) écrit : « in social democratic societies, the misrecognition, or revalorization of the indexical character of language may make discrimination on linguistic grounds publicly acceptable where the corresponding ethnic or racial discrimination is not ». La marchandisation et la déterritorialisation des voix des centres d'appel à des fins de productivité conduit à la production d'un double discours dans l'entreprise. Le management élabore une propagande d'unité et d'égalité entre les voix qu'elle produit. Puisqu'elle ignore les représentations et les stéréotypes qu'elles véhiculent en dépit des dispositifs de standardisation, elle permet aux mécanismes de discrimination de se mettre en place. Sans les déconstruire, elle les utilise pour instaurer et maintenir un rapport de force avec ses salariés français, en laissant planer la menace - même si elle s'en défend ${ }^{7}$ - d'une perte d'emplois sur le territoire national, et en justifiant l'exercice d'une forme de domination accrue sur ses salariés nord-africains (un renforcement du contrôle et de la surveillance, des temps de travail plus longs, des salaires plus faibles, etc.) par des motifs économiques. 
Cuisance, Marie Laure. « Ubiquité et discrimination des voix en centres d'appel : la quête illusoire et paradoxale d'un assureur en France et au Maghreb ». Nouvelle Revue Synergies Canada, № 15 (2022)

\section{Conclusion}

Avec la disparition des contacts de face-à-face au profit d'une gestion de la relation avec les clients en centres d'appel, la voix est devenue, pour l'entreprise, à la fois l'outil, la marchandise, la valeur et l'instrument de mesure de l'interaction avec ses clients, censée participer pleinement de l'efficacité du travail et de la rentabilité économique. L'enquête démontre la pertinence, dans ce contexte, de l'analyse socialement et spatialement située de la notion de voix, qui permet de prendre en compte les différents lieux dans lesquels elle s'inscrit et de décrire les représentations et les actes de l'ensemble des acteurs qui la produisent et l'écoutent. La description des contraintes techniques, verbales, spatiales et temporelles imposées à la voix des téléopérateurs, l'analyse des discours produits par le management, les salariés français, les clients sur ces voix et l'observation des ajustements mis en œuvre par les salariés nord-africains permettent de mettre en lumière des enjeux de domination, de différenciation et de discrimination peu visibles - donc difficiles à appréhender - car masqués par des justifications telles que le développement et la rentabilité économique ou la satisfaction du client.

Ces mécanismes de différenciation linguistique ont par ailleurs été analysés en linguistique socioculturelle (Bucholz et Hall, 2005) et plus récemment encore en raciolinguistique (Rosa et Flores, 2017), montrant le rôle que jouent les pratiques langagières dans les processus de différenciation sociale en lien aux questions de race et à l'histoire coloniale des société occidentales (Heller et Mc Elhinny, 2017). II n'aura pas échappé au lecteur qu'en faisant le choix d'un pays du Maghreb, l'entreprise a installé son centre d'appels dans un pays autrefois colonisé par la France. Au moment de l'enquête ethnographique, ses dirigeants avaient même envisagé de localiser une seconde plateforme dans un autre pays avant d'abandonner le projet, notamment du fait des craintes provoquées par la survenue du Printemps arabe. Deux possibilités avaient été étudiées, les deux destinations correspondant là encore à deux anciennes colonies françaises, et ce pour les mêmes raisons: la présence, sur le marché du travail, d'une " parole d'œuvre " francophone, formée aux métiers de la relation téléphonique et l'existence d'infrastructures adaptées à l'activité en plus de conditions légales et économiques favorables. Les données ethnographiques ne permettent pas, à ce jour, d'explorer les liens éventuels entre l'exploitation des voix nord-africaines par cette entreprise et l'histoire longue de la domination française sur les territoires où elles sont produites. II pourrait faire l'objet d'une prochaine enquête, au croisement entre une historiographie des relations de travail entre la France et ses anciennes colonies et une ethnographie des centres d'appel orientée par ce questionnement, pour tenter de faire apparaître la manière dont les mécanismes de différenciation se construisent ou se transforment à partir de la voix, ainsi que la façon dont les identités collectives, les altérités et les inégalités se reproduisent ou se reconfigurent dans le temps.

\section{Notes}

\footnotetext{
${ }^{1}$ Voir encadré.

2 Je me suis essentiellement intéressée aux interactions entre les clients et l'entreprise dans le cadre de l' " assurance habitation " mais la transformation de la relation concerne également les contrats (assurance automobile, etc.).
}

${ }^{3}$ Brevet de Technicien Supérieur, correspondant à deux années d'études après le Baccalauréat.

${ }^{4}$ Les salariés des centres d'appel de l'entreprise n'effectuent des appels dits "sortants » que lorsqu'il s'agit de rappeler un client pour l'informer de l'état de son dossier ou lui demander un complément d'information. Le travail ne comporte pas d'activité de démarchage téléphonique et les salariés n'ont de contact qu'avec des personnes déjà clientes de l'entreprise. De plus, ils effectuent également des activités dites de "back office " consistant à effectuer les tâches de gestion et de règlement des dossiers ( « sinistres ») en cours : demande de pièces justificatives, paiement des dédommagement, traitement des mails envoyés par les clients, des rapports d'expertise, etc. Je n'aborderai pas ici cette partie de leur activité qui sort du cadre de l'analyse de la voix.

\footnotetext{
${ }^{5}$ Tous les extraits cités ainsi au fil du texte sont issus du corpus de transcriptions des entretiens ethnographiques menés lors de mon enquête de terrain entre 2010 et 2012 dans le cadre de ma thèse de doctorat.
}

${ }^{6}$ Et ce, contrairement à d'autres situations en contexte français (Blanchet, 2013). 
Cuisance, Marie Laure. « Ubiquité et discrimination des voix en centres d'appel : la quête illusoire et paradoxale d'un assureur en France et au Maghreb ». Nouvelle Revue Synergies Canada, No 15 (2022)

\footnotetext{
${ }^{7}$ Au moment de l'enquête, malgré le nombre croissant de salariés embauchés au Maghreb, aucun licenciement n'avait eu lieu parmi les téléopérateurs français. Un vaste plan de départs volontaires était néanmoins en place à l'échelle de l'entreprise.
} 
Cuisance, Marie Laure. « Ubiquité et discrimination des voix en centres d'appel : la quête illusoire et paradoxale d'un assureur en France et au Maghreb ». Nouvelle Revue Synergies Canada, № 15 (2022)

\section{Bibliographie}

Arditty J., Blanchet P. (2008). La 'mauvaise langue' des 'ghettos linguistiques' : la Glottophobie française, une xénophobie qui s'ignore. ASYLONS, 3, http://terra.rezo.net/article748.html.

Blanchet P. (2013). Standardisation linguistique, glottophobie et prise de pouvoir. Dans M. Rispail (Dir.), Langues et pouvoirs, Cahiers de Linguistique 39(1), 93-108.

Boutet, J. (2001). La part langagière du travail. Langage \& Société, 98, 17-42.

Boutet, J. (2008). La vie verbale au travail: des manufactures aux centres d'appel. Toulouse, Octares.

Bucholz, M. et Hall K. (2005). Identity and interaction: A sociocultural linguistic approach. Discourse Studies, 7 (4-5), 585-614.

Buscatto, M. (2002). Les centres d'appels, usines modernes? Les rationalisations paradoxales de la relation téléphonique. Sociologie du travail, 1(44), 99-117.

Cowie C. (2007). The accents of outsourcing: the meanings of "neutral" in the Indian call centre industry. World Englishes, 26(3), 316-330.

Cuisance M.-L. (2016). Les petites catastrophes de la vie domestique. Ethnographie des mutations de la relation assureur-assurés chez Axa-France. Dilater l'espace, fragmenter le temps, intensifier la voix. [Thèse de doctorat en Ethnologie, Université Paris Ouest La Défense Nanterre]. Soutenue le 13 octobre 2016, 398 pages (dactyl.).

Duchêne A. (2011). Néolibéralisme, inégalités sociales et plurilinguisme: l'exploitation des ressources langagières et des locuteurs. Langage \& Société, 136(2), 81-108.

Duchêne, A. et Flubacher, M. C. (2015). Quand légitimité rime avec productivité: La parole-d'œuvre plurilingue dans l'industrie de la communication. Anthropologie et Sociétés, 39(3), 173-196.

Gadrey J. (2003). Socio-économie des services. La découverte.

Goffman, E. (1974). Les rites d'interaction. Éditions de Minuit.

Gumperz J.-J. (1989). Engager la conversation. Introduction à la sociolinguistique interactionnelle. Paris, Les Éditions de Minuit.

Habermas J. (1992). De l'éthique à la discussion. Le Cerf.

Heller M. (2003). Globalization, the New Economy and the Commodification of Language and Identity.Journal of Sociolinguistics, 7(4), 473-492.

Heller M. et Mc Elhinny B. (2017). Language, capitalism, colonialism. Toward a critical history. University of Toronto Press.

Kettani M. et M. Peraldi. (2011). Les mondes du travail. Segmentations et informalités. D'une Afrique à l'autre, migrations subsahariennes au Maroc, 55. http://www.un.org.ma/IMG/pdf/Segmentations_et_informalites.pdf. Consulté le 10 février 2021.

Rosa J. et Flores N. (2017). Unsettling race and language: Toward a raciolinguistic perspective. Language in Society, 46(5), 621-647.

Woolard K. (1998). Introduction : Langage ideology as a field of inquiry. Dans B. In Schiefflin, K. Woolard et Kroskrity (dir.), Language ideologies : Practice and Theory (p. 3-47). Oxford University Press. 